\title{
Cancer and Peripheral Deep Vein Thrombosis are both Independent Diseases
}

\author{
Elena Viktorovna Drozdova* \\ Cardiovascular Surgeon at the Samara Railway Clinical Hospital, Non-governmental Healthcare Institution, Russian Railways, Russia
}

*Corresponding author: Elena Viktorovna Drozdova, Cardiovascular Surgeon at the Samara Railway Clinical Hospital, Nongovernmental Healthcare Institution, Russian Railways, Russia

\begin{abstract}
There is an undisputed thesis: cancer has an often complication such as deep vein thrombosis. Cancer is the second leading cause of death globally and is responsible for an estimated 9.6 million deaths in 2018. (according to World Health Organization). Sickness rate of deep vein thrombosis is approximately 100 per 100000 population annually. Sickness rate of cancer in different countries is approximately 130-500 per 100000 population annually. Thus, if deep vein thrombosis is considered to be a complication of cancer, we must identify both these diseases simultaneously with frequency of 20-76.9\%.
\end{abstract}

a) The Research Objective: To determine whether peripheral vein thromboses are the complications of cancer.

b) The Method of the Research: Randomized prospective parallel controlled trial.

\section{Introduction}

In Russia there is a program according to which doctors should carry out the further survey of all the patients with deep vein thrombosis in order to identify cancer. Based on this program we conduct the following:
a) X-ray examination of the chest cavity,
b) Ultrasound diagnostics of the abdominal cavity organs,
c) Retroperitoneal space and genitourinary system
d) Fibrogastroduodenoscopy, fibrocolonoscopy,
e) Urologist/gynecologist examination;
f) Later, when the cancer is suspected-there is always in- depth follow-up.

Moreover, all the patients should visit a vascular surgeon again in 3 months and later in 6 months to control the dynamics of vein recanalization and the relevance of the extension of anticoagulant therapy. Thus, we can judge adequately about the presence or absence of suspicion of any tumor localization. However, having worked as a vascular surgeon for several years I have been wondering, why I have never detected cancer? So, I decided to analyze 100 cases of morbidity of deep vein thrombosis.

\section{Research Structure}

Primarily, 100 cases of morbidity of deep vein thromboses were analyzes. Thus, for the period 2011-2018 3 cases of cancer out of 100 cases of deep vein thrombosis were identified.

Patient A - cancer was identified in the oncological screening for the 1st time

Patient B - had a progression of cancer, was after ChT and with HIV- infection

Patient C - had metastatic pleurisy (T4b N3M1)

So, in this group the simultaneous rate of cancer and DVT is $3 \%$ instead of anticipated simultaneous sickness rate of $20 \%$ $76.9 \%$. Taking into account the rarity of cancer detection within the group of patients with deep thrombosis, I decided to select a control group of 100 people with cancer who were hospitalized for planned and urgent surgery to find out whether they had ever had deep vein thrombosis, any deviations in their coagulograms or whether they had some current problems with the post-thrombotic complications.

In group 2, I selected patients with cancer of maxillo-facial region, hepatobiliary system, large intestine, with and without 
progression of cancer; some of them were after chemotherapy and after radiation therapy. And to my great surprise in this, such a diverse group there were only 3 cases of the detection of deep vein thrombosis. All these three patients were of the age of 70 and over. Patient A (ICD 10 - C 25.0)-with simultaneous detection of cancer and acute deep vein thrombosis was an old woman who was hospitalized with the complications of cancer with metastasis in regional lymphatic and with a pulmonary thromboembolism. Patient B (ICD 10 - C 18.2) - with cancer and DTV in anamnesis, and he had been previously indicated in group 1 as a patient $\mathrm{A}$. Patient C (ICD 10 - C 34.0) - was with progression of cancer and deep thrombosis in anamnesis. Thus, we have: 2 cases (patients $A$ and B) with DVT in the group of patients with C 15-C 26 (according to ICD 10), and 1 case in the group C 30 - C 39 (according to ICD 10) - patient $C$ (Figure 1). There are 2 key arguments that cancer itself provides procoagulant activity they are: First - cancer has such often complication as deep vein thrombosis. Another one: there is a presence of fibrin and its decay products in the ascetic fluid.... Which could be considered as a result of hypercoagulation due to hyperfunction of ventral mesothelium. If we assume that deep vein thrombosis is not a cancer complication, we receive that all this deviation are the indicators of cancer as the disease itself. In this link we can note that we speak about only terminal stage of cancer! (Figure 2) Assuming that under the influence of carcinogens the response of the body occurs by the type of chronic autoimmune inflammation we receive the following chronic persistent phase: alteration, exudation and proliferation. Here is the graph which reflects the key aspects of pathogenesis of cancer-related coagulopathy (Figure 3). Since the first 2 stages occur mainly in the conditions of vasodilatation so they are not characterized by the active procoagulant process. But at the same time, they are characterized by the production of a large number of acute phase proteins which, of course, can potentiate some procoagulant activity but surely, they are not enough to be the cause of central or peripheral vein thrombosis. So fibrinogen, fibrin monomers and fibrin decay products in ascetic fluid most likely reflect chronic aseptic cancer-associated inflammatory process. Additionally we can also note that ascites itself is a marker of cancer decompensation, and it means that a very significant mechanism acts for its compensation-due to a toxic liver damage the synthesis of overwhelming number of coagulation factors is reduced and in the presence of dysfunction of vitamin K-dependent factors, hypocoagulation will prevail over hypercoagulation. As a result, it can be assumed that a significant pathology of the coagulant and anticoagulant systems of the body can occur mainly in the terminal stage of cancer by the type of chronic disseminated intravascular coagulation.

The frequency of single-stage occurrence of cancer and deep vein thromboses

I- Statistically expected the frecquency of simultaneous occurrence of morbidity of cancer and deep vein thromboses.

II- The obtained frecpuency of simultaneous occurrence of morbidity of cancer and deep vein thromboses.

III The frequency of simultaneous occurrence of cancer and deep vein thromboses.

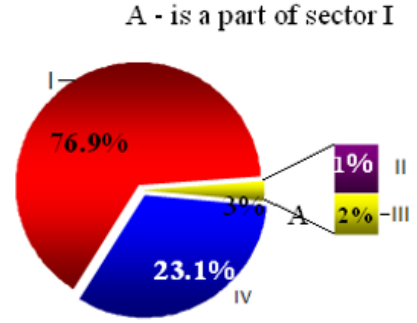

IV Statistically expected not simultaneous diseases

Figure 1.

The most probable reasons of coagulop athy

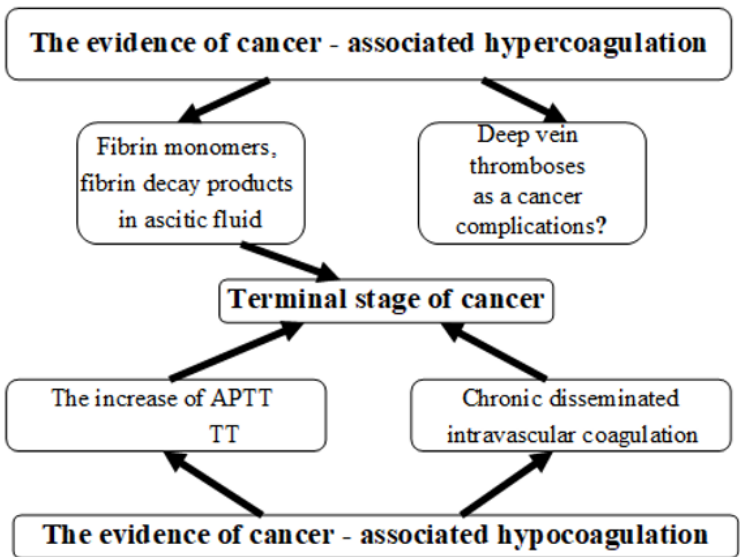

Figure 2. 


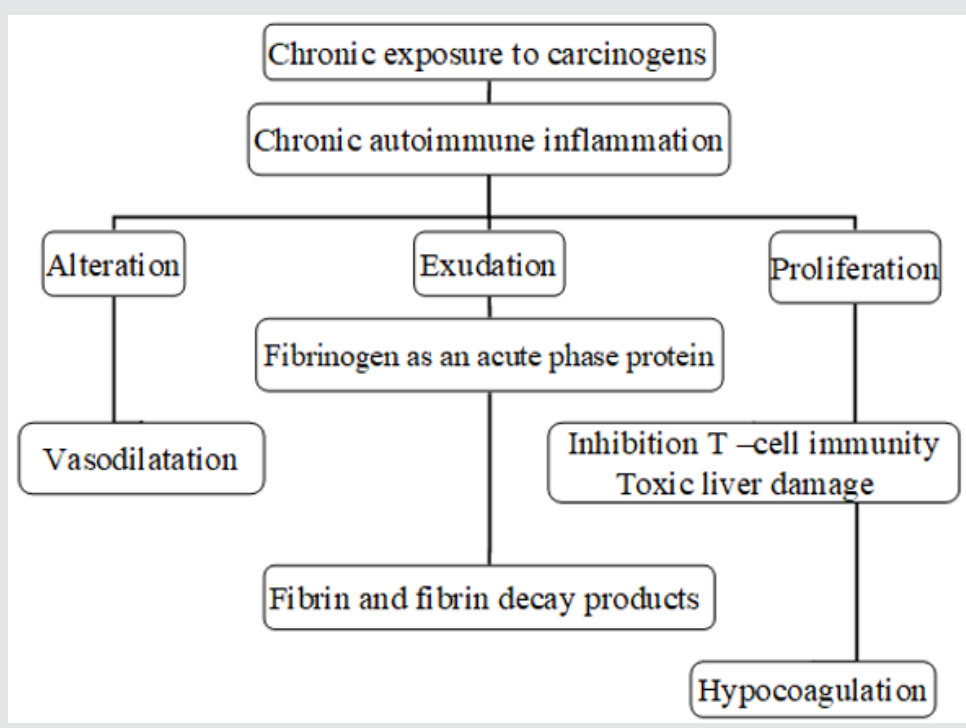

Figure 3.

\section{Results}

Assuming the equal probability of occurrence of both diseasescancer and deep vein thrombosis and experimental data, we can easily identify the following indicators which are commonly used in the evidence-based medical statistics.

\section{Bayes Factor}

In our case we suggest that we will not get even $50 \%$ cases of cancer from the group with deep vein thrombosis and that we will not get even $50 \%$ cases of deep vein thrombosis in the group with cancer. Thus, the Bayes factor is 0.03. Using Gaussian approximation $\mathrm{P}$ Value $=0.01$. This result can be considered as the evidence of significant strength.

\section{Conclusion}

a) We cannot argue that the key, trigger mechanism for starting such a disease as a deep vein thrombosis is cancer, and, so the deep vein thrombosis cannot be considered as a complication of cancer.

b) Patients with cancer decompensation most likely have changes in both coagulation and anticoagulation systems due to activation of tissue thromboplastins due to chronic aseptic inflammation and on the other hand because of reducing the production of blood coagulation factors due to toxic liver damage and intestinal dysfunction.
To Submit Your Article Click Here: Submit Article

DOI: $10.32474 /$ SCSOAJ.2019.03.000169

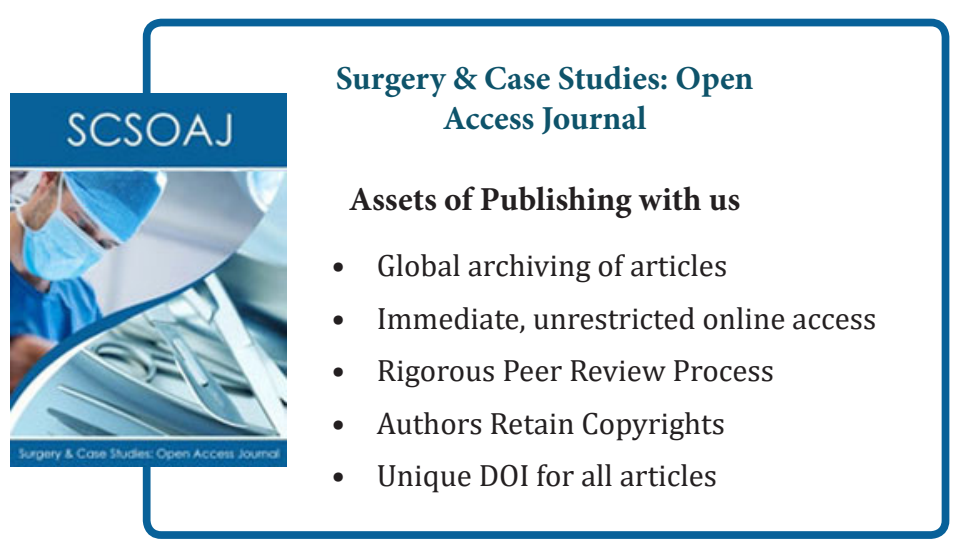

